\title{
Solid state electrical conductivity studies on manganese- molybdenum Schiff base polymer
}

\author{
S SUGANYA, FRANCIS P XAVIER* and K S NAGARAJA \\ Chemistry Department, *Physics Department, Loyola Institute of Frontier Energy, Loyola College, \\ Chennai 600034 , India
}

MS received 2 January 1998; revised 3 June 1998

\begin{abstract}
Heterobinuclear manganese-molybdenum complex with the Schiff base prepared by the condensation of diethylenetriamine with salicylaldehyde yielded a polymeric mixed metal coordination complex with the composition of $\left[\mathrm{Mn}_{1.5} \mathrm{MoO}_{3} \mathrm{~L}\right]$. The infrared spectral data indicated the presence of azomethine $(\mathrm{HC}=\mathrm{N})$ and Mo=O groups. ${ }^{1} \mathrm{H}$ NMR further confirmed the coordination of azomethine nitrogen and absence of phenolic hydrogen. The EPR studies suggested the presence of $M n(I I)$ in the complex. The solid state conductivity studies with the variation of potential and temperature indicated a specific conductivity of $1.6 \times 10^{-5} \mathrm{ohm}^{-1}$ $\mathrm{cm}^{-1}$. The activation energy was found to be $0.019 \mathrm{eV}$. Hall measurements indicate that the material is $p$-type which is further confirmed by iodine doping which increases the conductivity by two orders of magnitude.
\end{abstract}

Keywords. Schiff base polymer; conducting polymer.

\section{Introduction}

Coordination polymers are polymeric materials which contain chelated metal atoms in the structure. They are in general divided (Bailer 1961) into two types: (i) those in which the chelated metal atom (ion) is an integral part of the polymer framework; and (ii) those in which the metal atoms remain bound to the polymer having suitable donor groups in the framework. The current interest in coordination polymers comes largely from the fact that there is a growing need for polymers of greater stability and that, in many cases, organic materials should be stable against thermal or chemical interaction by coordination with metal ion (Banerjea 1994). Transition metal chelates derived from poly Schiff base have attained a prominent position (Carrober 1982) in the development of coordination chemistry of poly chelates. The poly chelates derived from the poly Schiff base obtained from 4,4'-dihydroxy-3,3'-diprophyldiphenyl with $o$-dianisidine were found to have decomposition temperature in the order $\mathrm{Ni}>\mathrm{Co}>\mathrm{Cu}>\mathrm{Mn}$ and thermal activation energy in the order $\dot{\mathrm{Ni}}>\mathrm{Cu}>\mathrm{Co}>\mathrm{Mn}$ (Patel and Jani 1986). The thermal stability of poly Schiff base is greater due to factors (Sutaria et al 1996) such as high molecular steric effect and resonance energy along the back bone unit. The poly Schiff base metal complexes prepared from diamines, salicylaldehyde and group II-V metal ions are found to contain channel like voids, which make them useful as chromatographic supports and absorbents

*Author for correspondence
(Patel and Patel 1989). The incorporation of an alternating aromatic molecules into a chain of metal ions as coordinating polymers produces materials which behave as semiconductors (Patel et al 1991). Thus, in the present study the coordination polymer prepared through interaction of $\mathrm{MnMoO}_{4}$ with the Schiff base due to interaction of diethylenetriamine and salicylaldehyde has been characterized and investigated for solid state semiconductivity.

\section{Synthesis of the sample}

Manganese(II) chloride $(0.71 \mathrm{~g}, 0.01 \mathrm{~mol})$ in water $\left(20 \mathrm{~cm}^{3}\right)$ was added to ammonium molybdate $(6.17 \mathrm{~g}$, $0.01 \mathrm{~mol})$ in water $\left(30 \mathrm{~cm}^{3}\right)$. The mixture was digested for $0.5 \mathrm{~h}$ over water bath up to $350 \mathrm{~K}$. The resulting pale yellow crystals of manganese molybdate (Venkataraman and Nagaraja 1996) were filtered off and dried in a desiccator.

Salicylaldehyde $(3.008 \mathrm{~g}, 0.01 \mathrm{~mol})$ was mixed with methanol $\left(10 \mathrm{~cm}^{3}\right)$, to which, diethylenetriamine $(2.954 \mathrm{~g}$, $0.01 \mathrm{~mol}$ ) was added. The reaction mixture was refluxed for $6 \mathrm{~h}$ to give the Schiff base.

To manganese molybdate $(0.254 \mathrm{~g})$, conc. $\mathrm{HCl}\left(5 \mathrm{~cm}^{3}\right)$ and $\mathrm{N}, \mathrm{N}^{1}$-dimethyl formamide $\left(20 \mathrm{~cm}^{3}\right)$ were added and digested over water bath for $0.5 \mathrm{~h}$. To the resulting mixture the Schiff base ligand solution was added slowly by gently stirring the mixture at $273 \mathrm{~K}$. The mixture was then transferred into a container over a large volume of ice water. A dark orange gummy substance was 
obtained. It was extracted with chloroform using a separating-funnel repeatedly. The aqueous layer was discarded. The resulting polymer along with chloroform and acetone $\left(10 \mathrm{~cm}^{3}\right)$ were left in china dish for 4 days so that chloroform could evaporate completely leaving behind dark yellow solid of Schiff base polymeric metal complex (figure 1).

Mo and Mn were analyzed by gravimetric (oxine) and EDTA volumetric method (Vogel 1978). TG analysis was carried out on Delta Series TGA-7 with a heating rate of $20 \mathrm{~K} \mathrm{~min}^{-1}$ (figure 2). IR spectra of the coordination polymer (figure 3 ) were recorded in the solid state on a Perkin-Elmer (model 983) in the region of 4000$40 \mathrm{~cm}^{-1}$ using $\mathrm{KBr}$ pellet. EPR spectrum was recorded in powder form at room temperature on a Varian E-4 $\mathrm{X}$-band spectrometer. ${ }^{1} \mathrm{H}$ NMR spectrum was recorded using a Hitachi R-600 high resolution spectrometer (figure 4).

\section{Experimental}

\subsection{Field dependent conductivity}

Pellets $\left(0.4 \times 0.4 \mathrm{~cm}^{2}\right)$ were made under a pressure of $10 \mathrm{MPa}$ in order to carry out electrical conductivity measurements. The pellet was fixed onto a pre-cleaned microscope glass plate. Thin copper wires (diam. $0.02 \mathrm{~cm}$ ) were fixed as electrodes onto the pellet using silver paint (with electrodes distance of about $2 \mathrm{~mm}$ ). The sample was then connected in series with a DC power supply (Aplab) and a picoammeter (Keithley 480). The applied field was increased in steps from 0 to $75 \mathrm{~V} / \mathrm{cm}$ and the corresponding current at room temperature was measured.

\subsection{Temperature dependent conductivity}

The pellet was then placed in the middle of a pyrex tube (diam. $5 \mathrm{~cm}$ ) with Kanthal wire (diam. $1 \mathrm{~mm}$ ) uniformly wound externally. The Kanthal wire-ends were

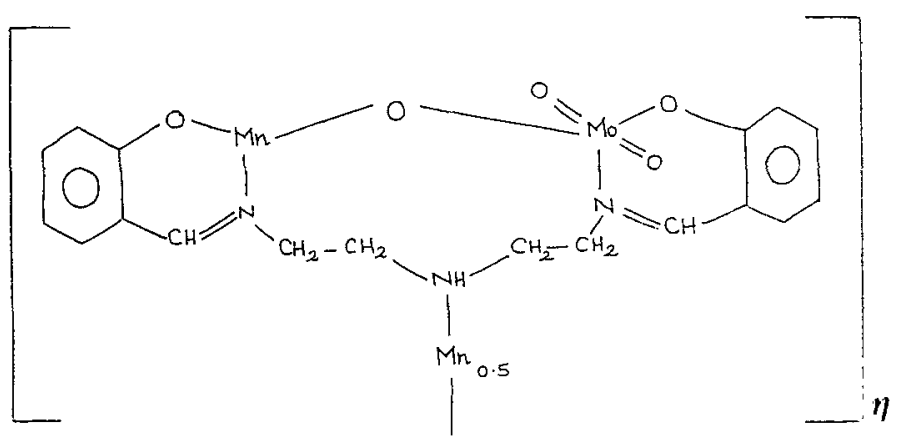

Figure 1. Proposed structure of coordination polymer $\left(\mathrm{Mn}_{1 \cdot 5} \mathrm{MoO}_{3} \mathrm{~L}\right)$. connected to a variac for temperature dependence studies. The pellet was placed in the middle region of the pyrex and the circuit mentioned above was made. A copperconstantin thermocouple kept at the pellet was used to measure the temperature of the sample. The current in the circuit with respect to varying applied field was measured keeping the temperature constant. Temperature was varied from $306 \mathrm{~K}$ to $376 \mathrm{~K}$ in steps of $10 \mathrm{~K}$. A graph with current vs applied field at various temperatures was plotted (figure 5). A plot of $\ln \sigma$ vs $1 / T$ is obtained to calculate the energy of activation (figure 6).

\subsection{Effect of halogen doping on conductivity}

The pellet was exposed to an atmosphere of iodine in a closed chamber for about $8 \mathrm{~h}$. Then the conductivity studies were carried out (current vs applied field) as mentioned above at time intervals of $1 \mathrm{~h}$. For a given field $(60 \mathrm{~V} / \mathrm{cm})$ the current was plotted as function of time (figure 7).

\subsection{Hall measurement}

Resistivity, carrier concentration and Hall mobility were measured by Hall measurement at room temperature (magnetic field strength being 500 gauss) on a pellet of thickness $0.24 \mathrm{~cm}$. The measurements were carried out using four-probe method (table 1).

\section{Results and discussion}

The elemental analysis of complex-polymer gave the $\mathrm{Mn}$ and $\mathrm{Mo}$ as $16 \%$ and $19.7 \%$ which corresponds to $\mathrm{Mn}_{1.5} \mathrm{MoO}_{3} \mathrm{~L}$ (figure 1). Thermogravimetric analysis in

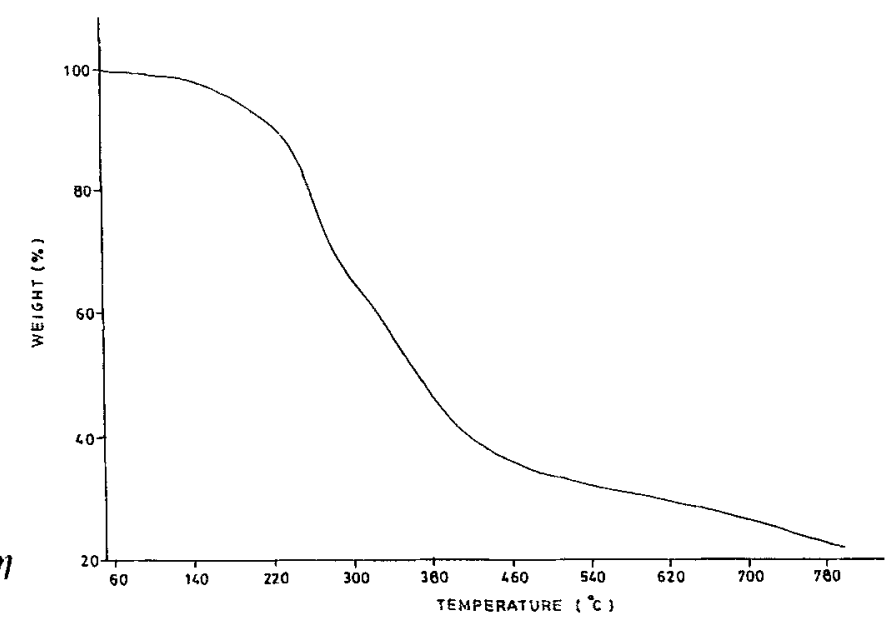

Figure 2. TG of manganese-molybdenum Schiff base polymer. 


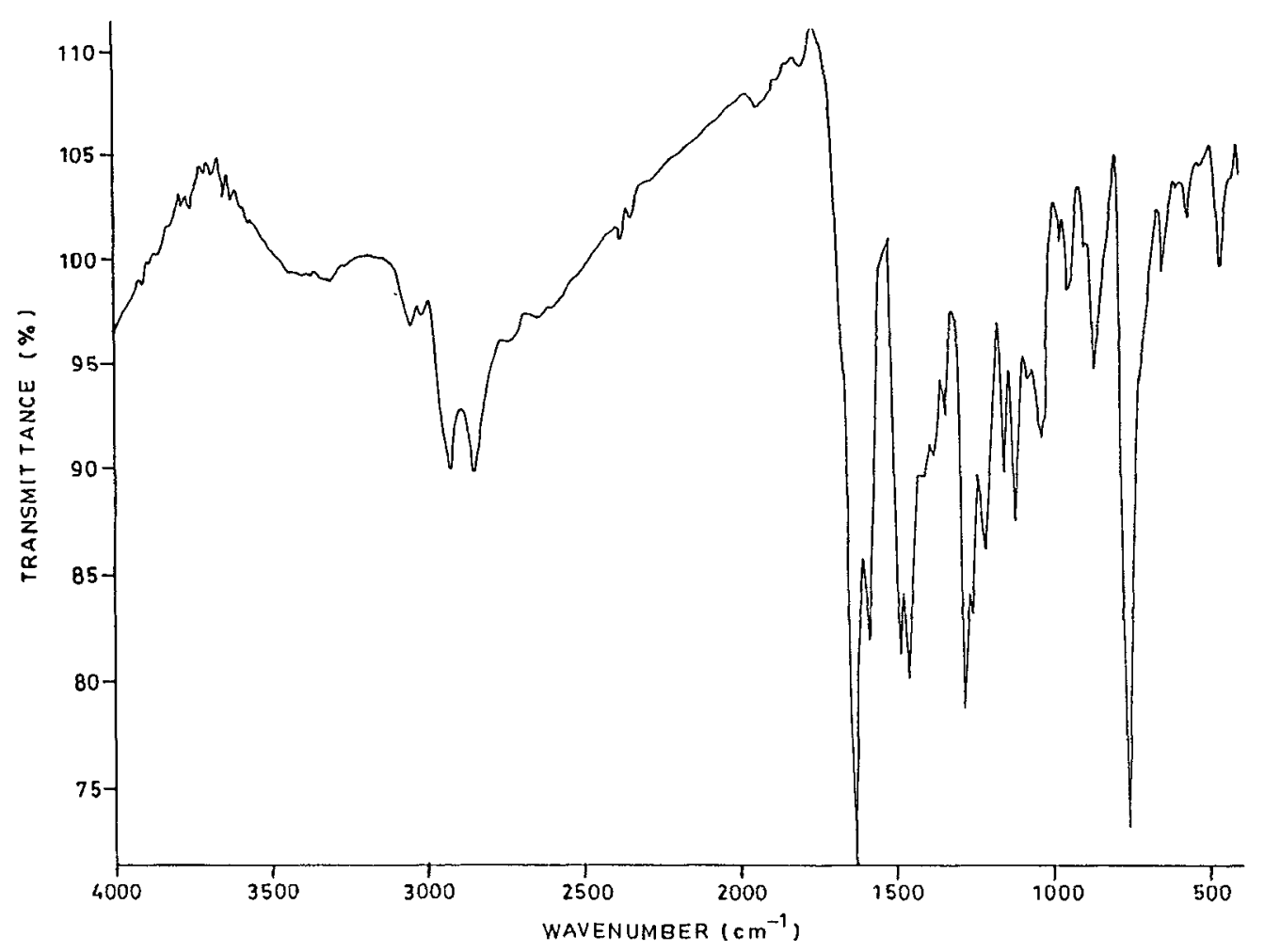

Figure 3. IR spectrum of manganese-molybdenum Schiff base polymer.

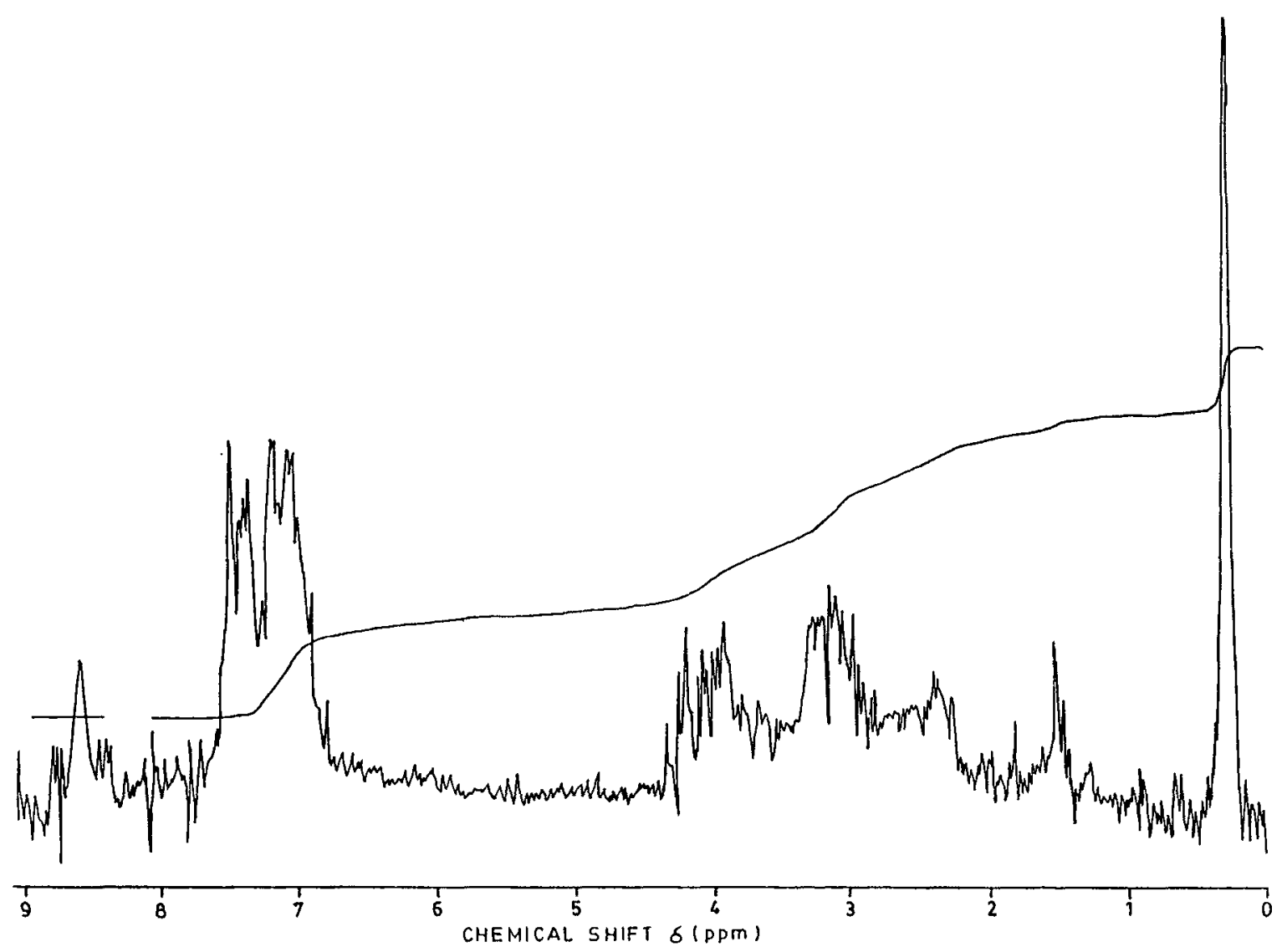

Figure 4. 'H NMR spectrum of manganese-molybdenum Schiff base polymer. 
air (figure 2) indicated a single step decomposition and absence of any coordinated or lattice water molecules. The decomposition occurs in the range $623 \mathrm{~K}-903 \mathrm{~K}$ to give $\mathrm{MnO}_{2}+\mathrm{MoO}_{3}$ as end residues as indicated by weight loss data from TG curve $(67.89 \%)$ which agrees with the calculated values $(67.57 \%)$ for the suggested composition. EPR spectrum of the complex has no signals due to $\operatorname{Mo}(V)$ centre but shows an ill defined fine-line pattern due to Kramer's degeneracy of $\mathrm{Mn}$ (II) centre. Thus the EPR spectra indicated the presence of $\mathrm{Mn}$ (II) and Mo(VI) in the complex (Drago 1976).

The IR spectrum of the complex (figure 3) indicated the absence of coordinated water molecule and the coordination due to azomethine $\left(\mathrm{C}=\mathrm{N}, 1610 \mathrm{~cm}^{-1}\right)$ and phenolic oxygen $\left(1100 \mathrm{~cm}^{-1}\right)$ (Sutaria et al 1996). A weak band at $900 \mathrm{~cm}^{-1}$ suggested a trans dioxo structure of molybdenum whereas a cis dioxo structure would

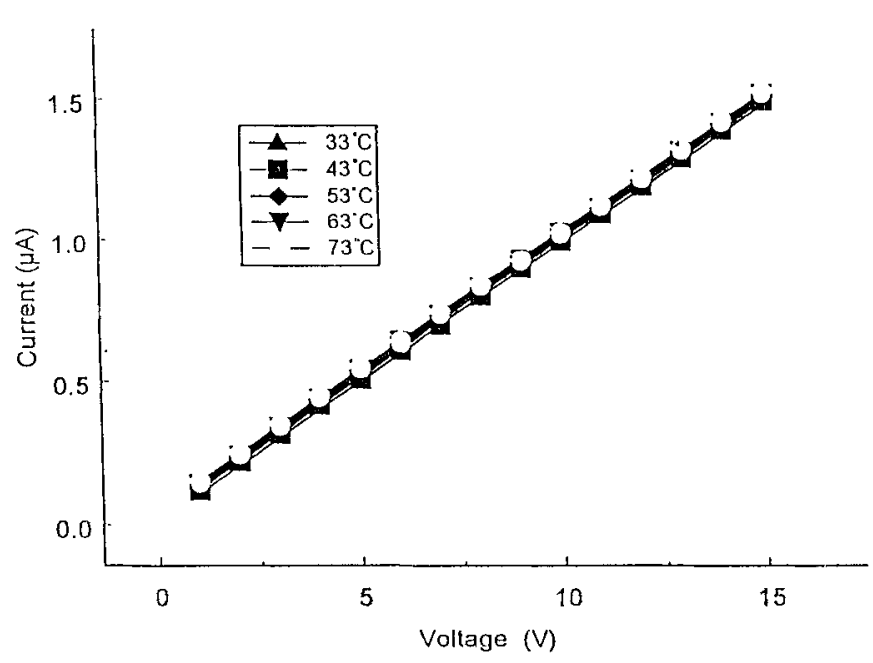

Figure 5. Temperature dependence of current vs voltage of manganese-molybdenum Schiff base polymer.

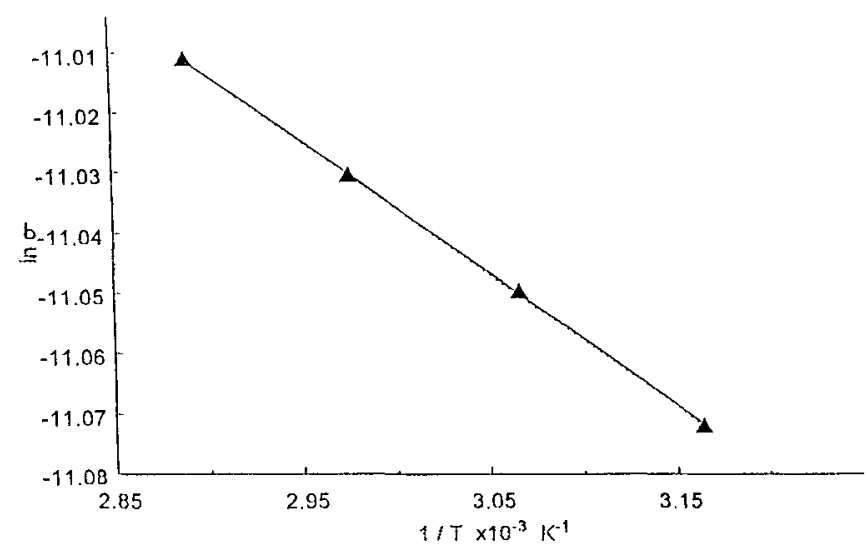

Figure 6. Specific conductivity vs $1 / T$ of manganese-molybdenum Schiff base polymer. have given a strong peak (Stifel 1977) around 875$1000 \mathrm{~cm}^{-1}$. The ligand geometry may force the molybdenum centre to assume a trans dioxo structure. ${ }^{1} \mathrm{H}$ NMR spectrum supported the coordination of azomethine nitrogen and presence of aromatic structure in the complex.

The current (conductivity) is linearly dependent on the applied field which indicates that the electrode contacts were ohmic. The linearity of current vs applied field does not vary on increasing the temperature and the magnitude of the current does not vary noticeably with increasing temperature. Above $346 \mathrm{~K}$ there is no variation with the temperature. However, from a plot of $\ln \sigma$ vs $1 /(k T)$ as shown in figure 6 , the activation energy, from the equation

$$
\sigma=\sigma_{0} \exp (-E / k T)
$$

is calculated $(0.019 \mathrm{eV})$. Since Hall measurements indicate that the majority charge carriers in the sample are of $p$-type, the conductivity is expected to increase in the presence of halogen dopant which is electron acceptor. In the present study doping the host complex with iodine increased the conductivity by two orders of magnitude (from specific conductivity of $1.48 \times 10^{-5} \mathrm{ohm}^{-1} \mathrm{~cm}^{-1}$ for the undoped sample to $6.80 \times 10^{-3} \mathrm{ohm}^{-1} \mathrm{~cm}^{-1}$ for the iodine doped sample). The conductivity of doped sample decreases exponentially with time as shown in figure 7 which is the expected result since iodine due to its high

Table 1. Hall measurement on manganese-molybdenum Schiff base polymer.

\begin{tabular}{ll}
\hline Resistivity & $4.893 \times 10^{8} \mathrm{ohm} \mathrm{cm}$ \\
Hall mobility & $409.5009 \mathrm{~cm}^{2} / \mathrm{Nec}$ \\
Carrier density & $3.49177 \times 10^{7} \mathrm{~cm}^{-3}$ \\
\hline
\end{tabular}

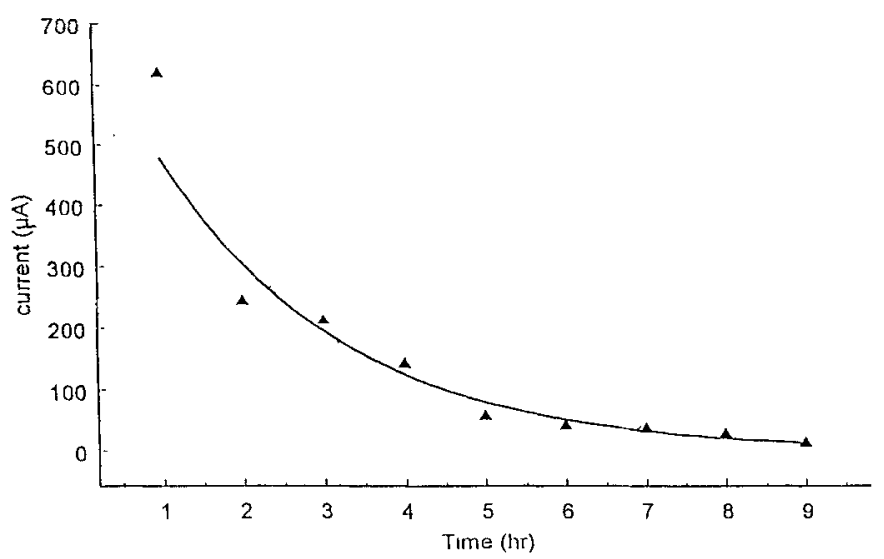

Figure 7. Current vs time of manganese-molybdenum Schiff base polymer doped with iodine at constant applied field $(E=66 \mathrm{~V} / \mathrm{cm})$. 
vapour pressure 'evaporates' with time and hence the exponential decrease in the iodine atoms correspondingly decreases the charge carrier and hence conductivity in the host sample decreases exponentially.

\section{Conclusion}

The structure of complex-polymer has been described as the manganese(II) atom and molybdenum(V) ion bridged by an oxo group with molybdenum having a trans dioxo structure along with the coordination of azomethine nitrogen and phenolic oxygen. The manganese ion is coordinated by a azomethine nitrogen and phenolic oxygen. Another manganese atom is bridged (figure 1) by the extension of a secondary nitrogen atom. Thus a continuous coordination polymer is obtained. The oligomeric nature observed during the preparation supports the polymeric nature rather than a discrete complex. The interesting feature is that the two hetero metal atoms are coordinated by the organic ligand. The conductivity investigation results in determination of activation energy and doping increases the conductivity substantially. Thus the complex as a rare example in the literature promises as a metallo organic compound for electrical conductivity.

\section{Acknowledgement}

One of the authors (SS) expresses thanks to Dr John Pragasam, Dr K Swaminathan, $\mathrm{Mr}$ A $\mathrm{R}$ Inigo and $\mathrm{Mr}$ Edwin Suresh for technical help and useful discussions.

\section{References}

Bailer J C Jr 1961 in Inorganic polymers (London: The Chemical Society) p. $61 \mathrm{ff}$

Banerjea D 1994 Coordination chemistry (Delhi: Tata-McGraw Hill) p. 148

Carrober C G Jr 1982 J. Macromol. Sc. Chem. 1717

Drago R S 1976 Physical methods in chemistry (Philadelphia: Saunders)

Patel M N and Jani B N 1986 J. Indian Chem. Soc. 63278

Patel V J and Patel M N 1989 Indian J. Chem. A28 428

Patel M N, Patel P P and Upadyaya D 1991 Indian J. Chem. A30 813

Stifel E I 1977 Prog. Inorg. Chem. 221

Sutaria D H, Patel J R and Patel M N 1996 J. Indian Chem. Soc. 1973

Venkataraman T and Nagaraja K S 1996 Indian J. Chem. A35 300

Vogel A I 1978 A text book of quantitative analysis (eds) J Bassett, R C Denney, G H Jeffrey and J Mendham (London: ELBS) 